\title{
FATORES CONDICIONANTES DA DESIGUALDADE SALARIAL NO BRASIL: ANÁLISE COM DADOS EM PAINEL PARA O PERÍODO DE 2007 A 2016
}

Matheus Oliveira de Alencar ${ }^{1}$ Jair Andrade Araújo²

Wellington Ribeiro Justo ${ }^{3}$

Resumo: Este estudo busca evidenciar os fatores condicionantes da desigualdade salarial nos municípios brasileiros nos anos de 2007 a 2016. Para alcançar esse objetivo, recorreu-se a variáveis municipais calculadas por meio dos microdados da RAIS/MTE. O método de análise empregado foi o de regressões com dados em painel. De maneira geral, as principais hipóteses do estudo foram confirmadas, já que se verificou, no Brasil, uma relação positiva entre desigualdade salarial e o tamanho do município, ou seja, constatou-se que municípios maiores, em termos populacionais, tendem a exibir maiores níveis de desigualdade. A mesma relação foi observada com o valor do salário médio do município. Em suma, foi possível observar que grande parte da desigualdade salarial é gerada pela existência dos diferenciais de salário entre setores de trabalho, classe de escolaridade e sexo do trabalhador.

Palavras-chave: Desigualdade. Salários. População. Municípios. Brasil.

\section{CONDITIONING FACTORS OF WAGE INEQUALITY IN BRAZIL: PANEL DATA ANALYSIS FOR THE PERIOD OF 2007 TO 2016}

Abstract: This study seeks to expose the conditioning factors of wage inequality in Brazilian municipalities between 2007 and 2016. To reach this objective, municipal variables were calculated using micro data from the RAIS/MTE. The analysis method used were regressions with panel data. In general, the main study hypotheses were confirmed, since it was verified that in Brazil a positive relation between wage inequality and municipality size, that is, it was verified that larger municipalities, in population terms, tend to present higher inequality levels. The same relationship was observed on municipality average salary. To summarize, it can be observed that a large part of the wage inequality is generated by the existence of wage differentials between labour sectors, education level and worker's sex.

Keywords: Inequality. Wage. Population. Municipalities. Brazil.

\section{FACTORES CONDICIONANTES DE LA DESIGUALDAD SALARIAL EN BRASIL: ANÁLISIS CON DATOS EN PANEL PARA EL PERÍODO 2007 A 2016}

Resumen: Este estudio busca evidenciar los factores condicionantes de la desigualdad salarial en los municipios brasileños en los años de 2007 a 2016. Para alcanzar ese objetivo, se recurrió a variables municipales calculadas por medio de

\footnotetext{
${ }^{1}$ Universidade Federal do Rio Grande do Sul (UFRGS), Departamento de Economia e Relações Internacionais, Porto Alegre (RS), Brasil, matheusalencar29@gmail.com, https://orcid.org/0000-00029594-6354.

2 Universidade Federal do Ceará (UFC), Departamento de Economia Agrícola, Fortaleza (CE), Brasil, jaraujoce@gmail.com, https://orcid.org/0000-0001-6297-9558.

3 Universidade Regional do Cariri (URCA), Departamento de Economia, Crato (CE), Brasil, justowr@yahoo.com.br, https://orcid.org/0000-0002-4182-4466.
} 
los microdatos de la RAIS/MTE. El método de análisis empleado fue el de regresiones con datos en panel. En general, las principales hipótesis del estudio fueron confirmadas, ya que se verificó, en Brasil, una relación positiva entre desigualdad salarial y el tamaño del municipio, o sea, se constató que municipios mayores, en términos poblacionales, tienden a exhibir mayores niveles de desigualdad. La misma relación fue observada con el valor del salario medio del municipio. En suma, fue posible observar que gran parte de la desigualdad salarial es generada por la existencia de los diferenciales de salario entre sectores de trabajo, clase de escolaridad y sexo del trabajador.

Palabras clave: Desigualdad. Salarios. Población. Municipios. Brasil.

\section{Introdução}

O estudo das questões que envolvem a desigualdade salarial detém grande importância nas discussões acadêmicas, tanto no plano nacional como no contexto internacional. Analisar a desigualdade de rendimentos do trabalho é essencial para se entender a dinâmica da desigualdade de renda, de maneira geral.

No Brasil existem vários estudos que se reportam a essa temática, a maioria dos quais se concentra num exame macrogeográfico. Estudar a desigualdade no nível de cidades, entretanto, também é fundamental, pois, segundo Lee et al. (2016), seus efeitos podem ser sentidos mais intensamente nas cidades. Glaeser et al. (2009) também reforçam a necessidade de se estudar a desigualdade no contexto local e assinalam que, em cidades mais desiguais, podem ser notados maior taxa de criminalidade, menor bem-estar social, maior mobilidade populacional e uma associação negativa com o crescimento da renda.

De modo geral, no Brasil, distintos estudos apontaram significante queda na desigualdade salarial desde o início dos anos 2000. Barros et al. (2007) acentuam que no período 2001-2004, ocorreu acentuada redução nos níveis de desigualdade de renda no País, incluindo-se nessa categoria os rendimentos salariais ${ }^{4}$. No entanto, afirma também que, apesar de tais avanços, o Brasil ainda se encontra entre os países mais desiguais do mundo.

A associação entre a desigualdade salarial e o tamanho da cidade é uma relação pertinentemente estudada nos últimos anos em vários países. Baum-Snow e Pavan (2013) demonstraram a existência de uma relação positiva entre a desigualdade salarial e o tamanho das cidades dos Estados Unidos no período 1979-2007, quando foi observado um crescimento mais rápido da desigualdade,

\footnotetext{
${ }^{4}$ Cerca de $76 \%$ da renda das famílias brasileiras advêm do trabalho. Estima-se que grande parte da desigualdade de renda entre famílias resulta das disparidades de remuneração reveladas pelo mercado de trabalho (LAVINAS; NICOLL, 2006).
} 
principalmente em grupos bem capacitados em cidades maiores. Ainda nos Estados Unidos, Behrens e Nicould (2008), recorrendo a regressões quantílicas, também demonstraram que cidades maiores e, consequentemente, que possuem mais trabalhadores, são tanto mais produtivas quanto mais desiguais e polarizadas ${ }^{5}$ no que se refere à distribuição salarial, além de evidenciar que o comércio entre cidades está associado a maiores níveis de desigualdades.

Ao buscar evidenciar os determinantes da desigualdade salarial nos mercados de trabalho locais suecos, Korpi (2007) observou relação positiva entre a desigualdade salarial e o tamanho da população local. Lee et al. (2016), ao utilizar o número de empregados como proxy para o tamanho da população, observaram a mesma relação nas cidades britânicas. Esses autores ainda destacaram que cidades com salário médio mais elevado, tendem também a apresentar maior desigualdade de salários.

Em razão do que foi exposto, surgem os seguintes questionamentos: que fatores influenciam o nível de desigualdade salarial dos municípios brasileiros? No Brasil, municípios maiores em termos populacionais tendem a ser mais desiguais? Qual a relação entre o valor do salário médio e o grau de desigualdade salarial dos municípios brasileiros?

Nesse contexto, este estudo tem como objetivo evidenciar os condicionantes da desigualdade salarial nos municípios brasileiros no intervalo de 2007 a 2016 . Além disso, se intenta comparar o Brasil a outros países específicos no tocante ao comportamento da desigualdade salarial, por meio de estudos internacionais já realizados.

Apesar deste estudo abordar um assunto amplamente discutido na esfera econômica, ele avança em termos de literatura, ao melhorar o entendimento acerca das causas da desigualdade salarial no Brasil e preenchendo algumas lacunas, como: examinar a desigualdade salarial no Brasil em escala municipal; acrescentar variáveis importantes para o controle da desigualdade de salários; bem como trabalhar com dados mais recentes.

Outra contribuição importante do estudo ora relatado, além da análise na escala municipal, é analisar a relação entre tamanho populacional do município e

\footnotetext{
${ }^{5}$ A polarização, neste caso, como o próprio nome já induz, caracteriza-se como uma medida de desigualdade de renda em que se verifica o nível de concentração de população empregada recebendo maiores e menores salários, ou seja, a polarização dessa população na distribuição salarial. A teoria das medidas de polarização foi desenvolvida para o caso em que a distribuição de renda pode ser descrita usando funções de densidade (DUCLOS et al. 2004).
} 
valor do salário médio com a desigualdade salarial, uma vez que não foram encontradas pesquisas que fizessem tal análise para o Brasil.

Em adição a este texto introdutório, o artigo é composto por mais quatro seções, a segunda das quais se refere a vários estudos sobre desigualdade de salários realizados em diversos países, alguns realizados para o Brasil. No terceiro segmento, são descritos os procedimentos metodológicos, na quarta trazem-se os resultados e, por fim, na quinta seção, estão as considerações finais.

\section{Revisão de literatura}

$\mathrm{Na}$ literatura é consenso o fato de que a desigualdade de salários traz uma série de consequências negativas para a sociedade como um todo, e não apenas para as classes com rendimentos mais baixos. Aumento da criminalidade, piores condições de saúde, diminuição do bem-estar social, entre outros aspectos sociais negativos, são, em parte, implicações de um maior grau de desigualdade de renda e, por analogia, de desigualdade salarial (SZWARCWALD et al., 1999; RESENDE; ANDRADE, 2011; MARQUES JUNIOR, 2014).

$\mathrm{Na}$ literatura internacional, no que se refere aos fatores condicionantes da desigualdade de salários no plano local, destacam-se estudos como os de Korpi (2007), que estudou a relação da desigualdade no mercado de trabalho sueco com o tamanho da população da cidade, Glaeser et al. (2009), que analisam as causas e consequências da desigualdade nas cidades dos Estados Unidos e Lee et al. (2016), que investigam os padrões e níveis da desigualdade nas cidades britânicas e os seus determinantes.

Na Suécia, Korpi (2007) observou relação positiva entre a desigualdade salarial nos mercados de trabalho locais e o tamanho das suas respectivas populações, bem como uma relação negativa entre a desigualdade salarial e a taxa de emprego. No Reino Unido, Lee et al. (2016), utilizando o número de empregados como proxy para o tamanho da população das cidades, observaram que esta variável tem influência positiva na desigualdade de salários, ou seja, as cidades maiores tendem a ser mais desiguais. Ainda destacam que, apesar de mais desiguais, as cidades britânicas não são necessariamente mais polarizadas quanto à distribuição de salários.

O nível de desenvolvimento econômico e a desigualdade salarial, também, constituem uma relação amplamente defendida por pesquisadores de vários países. Kuznets (1955) sugeriu que o crescimento econômico expressa dois efeitos sobre a 
desigualdade, dado que, a priori, ocorre um aumento desta, em virtude do baixo número de pessoas trabalhando em setores com salários mais elevados. No segundo momento, entretanto, com o gradual aumento de trabalhadores empregados nesses setores, a desigualdade tende a diminuir. Glaeser et al. (2009) e Lee et al. (2016) observaram que cidades com salário médio mais elevado, tendem também a apresentar maior desigualdade de salários.

Outro fator visto como um dos principais condicionantes da desigualdade de salários está relacionado ao conhecimento e habilidades da pessoa para desenvolver determinado trabalho, ou seja, capital humano. A distribuição do nível de capital humano, principalmente em escala local, possui grande influência na condição de desigualdade de determinado lugar. Segundo Lee et al. (2016), o fato de haver trabalhadores mais qualificados e mais bem remunerados desencadeia a desigualdade, ocorrendo esse fenômeno especialmente nas maiores cidades.

Há de se considerar, no entanto, o fato de que a escolaridade pode expressar efeitos distintos sobre o nível de desigualdade. Lemieux (2006) sugere que a maior parte de um aumento da desigualdade salarial decorre do aumento dos retornos ao ensino pós-secundário, ou seja, as mudanças na desigualdade salarial estão cada vez mais concentradas no topo da distribuição salarial.

Budría e Telhado-Pereira (2011), ao investigarem a conexão entre educação e desigualdade salarial em nove países europeus, concluíram que trabalhadores com nível superior denotam maior dispersão salarial quando comparados a trabalhadores com menor nível educacional. Sugerem, também, que uma expansão educacional do ensino superior pode aumentar a desigualdade salarial global na Europa. Destacam, ainda, que o efeito da educação na desigualdade salarial pode expressar natureza diferente de um país para outro, e, assim, observaram três grupos distintos de países nessa pesquisa. No primeiro, composto por Grécia, Noruega e Itália, a educação contribuiu para o aumento da desigualdade salarial, ao passo que, no segundo grupo, Alemanha, Reino Unido, França e Finlândia, a influência da educação sobre a desigualdade salarial foi ambígua. No terceiro grupo, Portugal e Suécia, a desigualdade diminuiu com o aumento da educação.

De maneira geral, Budría e Telhado-Pereira (2011) constataram que, na Europa, tem havido uma tendência para a dispersão salarial no grupo de pessoas com melhor qualificação, seja pelo maior nível de escolaridade, seja pelo maior grau de experiência, o que favorece a elevação da desigualdade salarial nesse território. 
Existem também pesquisas que analisam a influência da participação da força de trabalho de alguns setores da economia na desigualdade salarial. Lee et al. (2016) verificaram associação negativa entre participação da força de trabalho no setor público e a desigualdade salarial, ou seja, em cidades com maior participação do setor público, os salários tendem a ser mais iguais. Já no que se refere à participação da força de trabalho no setor financeiro, observou fraca relação positiva entre esta variável e o grau de polarização da cidade.

No que se refere à influência do grau de participação de trabalhadores do sexo masculino e de trabalhadores estrangeiros no mercado de trabalho, Korpi (2007) constatou que tais variáveis não apresentaram importância para explicar a desigualdade salarial nas cidades suecas, sendo que a participação de trabalhadores estrangeiros ainda apresentou significância em uma das medidas de desigualdade utilizadas no estudo, porém com efeito muito pequeno. Lee et al. (2016) observaram influência negativa da participação de trabalhadores estrangeiros sobre duas das medidas de desigualdade empregadas em seu estudo para o Reino Unido, no entanto, também consideram trivial, esse efeito, uma vez que só era observado quando se incluía a cidade de Londres na amostra.

Nacionalmente, mencionam-se estudos como os de Barros e Reis (1991), IPEA (2006), Ramos (2007), Ferreira et al. (2008), Vaz e Hoffmann (2007) e Miro et al. (2016), que analisam as causas e o processo da desigualdade de renda e salários.

A desigualdade de salários no mercado de trabalho ocorre desde o momento em que se remunera de modo diferenciado trabalhadores com o mesmo potencial produtivo (IPEA, 2006). Vários estudos, com ênfase para Barros e Reis (1991), Ramos (2007) e Ferreira et al. (2008), apontam que apenas o nível de escolaridade e a experiência dos trabalhadores já representam expressiva parte da desigualdade salarial no Brasil.

Ao investigar as causas da diminuição da desigualdade salarial no Brasil, no período 1995-2014, Miro et al. (2016) observaram que, com relação ao efeito quantidade, isoladamente, o aumento educacional da força de trabalho exprimiu efeitos favoráveis à desigualdade salarial, contribuindo com a concentração de rendimentos, principalmente de 2004 a 2014. Os autores associam esse resultado 
ao fenômeno "paradoxo do progresso"6. Esse comportamento também já havia sido constatado por Ferreira et al. (2008), no Brasil, no período de 1981 a 2004. Segundo IPEA (2006), a expansão educacional apenas será eficiente no combate à desigualdade se ocorrer em consonância com o aumento da qualidade da educação.

Além disso, Miro et al. (2014) também verificaram que, para a faixa de pessoas que possuem menor grau de escolaridade, um aumento na escolaridade enseja efeito de desconcentração dos salários. Já para a faixa de pessoas com escolaridade mais elevada, os efeitos são favoráveis à desigualdade salarial.

No entendimento de Hoffmann (2001), apesar da divergência teórica acerca dos efeitos da educação sobre a desigualdade de salários, o aumento da escolaridade é um objetivo para o país, e há um consenso no que se refere à necessidade do aumento da escolaridade no Brasil.

A idade do trabalhador também representa parâmetro que pode influenciar na remuneração do trabalho. Em geral, estudos como os de Chaves (2002), Balassiano et al. (2005) e Santos (2011) apontam que o auge do rendimento do trabalhador é na idade de 30 até 49 anos, nos 50 anos, começa o arrefecimento no salário, o que pode variar de acordo com sexo e o período analisado.

No Brasil, a influência da participação dos trabalhadores do setor público no mercado de trabalho sobre a desigualdade tende a ser positiva, uma vez que, embora contribua com a equalização dos salários entre as distintas classes de funcionários públicos, no que se refere a características como sexo e raça, quando comparado com trabalhadores do setor privado que possuem atributos semelhantes, os salários do setor público são significativamente superiores (VAZ; ROFFMANN, 2006; SOUZA; MEDEIROS, 2013).

Outro aspecto essencial a ser considerado como influente na desigualdade salarial é a discriminação por sexo. Assim como é observado em todos os países, no Brasil, a remuneração de trabalhadores do sexo masculino é significativamente superior à remuneração das mulheres (LAVINAS; NICOLL, 2006; IPEA, 2006).

Não se encontrou na literatura nacional nenhum tipo de estudo de teor quantitativo que analisa a relação entre tamanho da cidade, salário médio, proporção de trabalhadores do setor financeiro e proporção de trabalhadores estrangeiros com a desigualdade salarial, sendo, pois, uma contribuição deste estudo.

\footnotetext{
6 O "paradoxo do progresso", discutido por Bourguignon et al. (2005), é um fenômeno caracterizado por uma situação em que a ocorrência de progresso em termos educacionais pode causar o aumento da desigualdade de renda.
} 


\section{Metodologia}

\section{Base de dados}

A base de dados utilizada neste estudo foi obtida por meio dos microdados da Relação Anual de Informações Sociais (RAIS), vinculada ao Ministério do Trabalho, para os municípios brasileiros, compreendendo os anos de 2007 a 2016. Esse recorte temporal coincide com o início do segundo mandato do governo Lula até os primeiros meses do governo Temer.

Apesar da limitação dessa fonte de dados, uma vez que capta apenas informações por vínculo de emprego formal, ela ainda é bastante satisfatória, haja vista a amplitude de tais informações no que se refere às características do trabalhador. Além disso, possibilita informações municipais, haja vista que outra fonte que fornecesse tais variáveis poderia ser o censo demográfico, no entanto, 0 último se refere ao ano de 2010.

A variável dependente empregada na análise foi o índice de desigualdade de Gini, calculado por meio do salário médio anual de cada trabalhador do município. $\mathrm{O}$ índice de Gini é uma das medidas de desigualdade mais utilizadas e difundidas em estudos que analisam desigualdade de renda ou salários, e configura-se como um eficiente método para o cálculo do grau relativo de desigualdade com base na curva de Lorenz. O coeficiente de Gini varia de zero a um, sendo que, quanto mais próximo de zero, menor a desigualdade e, logicamente, quanto mais perto de um, maior a desigualdade (HOFFMANN, 2006).

Para explicar o comportamento da desigualdade salarial nos municípios brasileiros, foram aplicadas, no modelo, como controle, variáveis municipais construídas por meio de características socioeconômicas dos trabalhadores que compõem a população do município. As variáveis explicativas municipais empregadas na análise foram: o número de vínculos de trabalho ativos; o salário médio; a taxa de trabalhadores no setor financeiro; a taxa de trabalhadores com nível superior; a taxa de trabalhadores estrangeiros; a taxa de ganhadores de pico; e a taxa de trabalhadores do sexo masculino.

Assim como em Lee et al. (2016), o número de vínculos ativos foi utilizado como proxy para o tamanho do município. Com base na literatura, supõe-se que, quanto maior for o tamanho do município, maiores serão as diferenças entre os 
trabalhadores que o compõem e, consequentemente, maior seu nível de desigualdade.

Já o salário médio municipal, obtido por meio da esperança do salário médio anual de cada trabalhador do município, é utilizado como uma medida de riqueza. Espera-se observar relação positiva entre o valor do salário médio e a desigualdade salarial, assim como foi verificado por Korpi (2007), Glaeser et al. (2009) e Lee et al. (2016).

Para averiguar a possível relação entre serviços financeiros e a desigualdade salarial, controlou-se pela taxa de trabalhadores no setor financeiro no município, calculada como a participação dos trabalhadores empregados no setor de atividades financeiras e de seguros. Como observado em outros países, a expectativa é de se constatar relação direta, ou seja, quanto maior a participação de trabalhadores no setor financeiro, maior o nível de desigualdade salarial do município.

Quanto à taxa de trabalhadores no setor público no município, também se espera que, quanto maior a participação destes trabalhadores no mercado de trabalho, maior será o grau de desigualdade de salários, uma vez que o setor público tende a remunerar melhor do que o setor privado.

A taxa de trabalhadores com nível superior também foi utilizada como controle neste estudo. Essa variável foi utilizada para captar o efeito do estoque de capital humano sobre a desigualdade de salários. Como, no Brasil, a parcela da população que possui nível superior ainda é pequena, comparativamente a outros países, um crescimento até certo ponto nessa proporção tende a aumentar a desigualdade salarial, ao contribuir com a obtenção de salários mais elevados por estes trabalhadores mais qualificados.

No que se refere à taxa de trabalhadores estrangeiros, não há um consenso na literatura quanto ao seu efeito sobre o nível de desigualdade salarial, pois tal efeito varia de um país para outro. Como, no entanto, a proporção de trabalhadores estrangeiros nos municípios brasileiros é bastante pequena, é possível que esta variável não exprima grande influência sobre a desigualdade.

A taxa de "ganhadores de pico" refere-se à proporção de trabalhadores com idade de 42 a 56 anos, pois os salários no Brasil atingem o pico nessa faixa de idade e, assim, se espera que contribua positivamente com a desigualdade de salários ${ }^{7}$.

\footnotetext{
7 O cálculo dessa faixa de idade (42 a 46 anos) foi realizado por meio de uma equação minceriana, utilizando dados da Pesquisa Nacional de Amostra por Domicílio (PNAD) do ano de 2014. A escolha desse ano para o cálculo justifica-se pela então estabilidade no mercado de trabalho, com taxas de desocupação bastante baixas naquele momento.
} 
Por fim, no que diz respeito à taxa de trabalhadores do sexo masculino, estudos como os de Miro e Suliano (2010), Batista e Cacciamali (2009) e Assis e Alves (2014), apontam haver diferencial de salários entre trabalhadores do sexo masculino e do sexo feminino no Brasil, o que tem efeito negativo sobre a distribuição salarial e, consequentemente, contribui com o aumento da desigualdade de salários. Com efeito, pressupõe-se que, quanto maior a proporção de trabalhadores do sexo masculino, maior o grau de desigualdade salarial do município.

\section{Dados em painel}

Para a estimação e análise das correlações entre a desigualdade de salários (índice de Gini) e seus condicionantes, foram empregados modelos de regressão linear múltipla com dados em painel, genericamente tratados como dados longitudinais. Esse tipo de modelo é caracterizado por exprimir observações em duas dimensões, geralmente, tempo e espaço, ou seja, consistem em observações de corte transversal (cross section) referentes a períodos distintos (SONAGLIO et al. 2010). No caso deste estudo, as unidades observacionais são os municípios brasileiros $^{8}$ e o período compreende os anos de 2007 a 2016.

Dentre as vantagens de se utilizar dados em painel, pode-se mencionar a disposição de informações que proporcionam melhor investigação no que tange à dinâmica de mudança nas variáveis, permitindo considerar o efeito das variáveis não observadas. Além disso, há de se salientar que, em razão do maior número de graus de liberdade, menor colinearidade e maior variabilidade da amostra, em comparação com dados de corte transversal ou de séries temporais, há melhor inferência nos parâmetros estimados, ou seja, melhor eficiência dos estimadores econométricos (GREENE, 2000; BALTAGI, 2005; HSIAO, 2003).

Ainda segundo Wooldridge (2002), existem duas possibilidades para a modelagem dos efeitos não observados, o método de efeitos fixos e o de efeitos aleatórios, sendo, a escolha do modelo mais adequado, fundamentada no próprio comportamento dos efeitos não observados.

Em modelos de regressão com dados em painel, o distúrbio é composto por dois elementos: o efeito individual inerente às unidades observáveis (neste caso, os

\footnotetext{
${ }^{8}$ Segundo o IBGE (2018), o Brasil possui 5.570 municípios. Para garantir a qualidade da análise, foram excluídos da amostra municípios que possuíam um número de observações menor do que dez. Também foram excluídos municípios que não tinham informações para todos os anos analisados, a fim de tornar o painel balanceado. Sendo assim, foram considerados neste estudo 5.555 municípios.
} 
municípios) e o distúrbio estocástico. O primeiro pode ou não ser correlacionado com alguma variável explicativa do modelo (HSIAO, 2003; MACHADO et al. 2005).

Quando houver correlação entre o efeito individual e algum regressor do modelo, a estimação deve ser feita por meio do estimador de efeitos fixos, permitindo o controle dessa correlação. Já no caso de o efeito individual ser puramente aleatório e não correlacionado com qualquer variável explicativa, o estimador de efeitos aleatórios é o mais apropriado (HSIAO, 2003; SONAGLIO et al. 2010).

A determinação da ocorrência de correlação entre o efeito individual e os regressores do modelo pode ser feita por meio da aplicação do Teste de Hausman. O Teste de Hausman é procedimento mais utilizado para auxiliar na determinação do modelo mais adequado. Consiste, basicamente, em uma comparação estatística entre o vetor de estimativas de efeitos fixos e o vetor de estimativas de efeitos aleatórios para o mesmo modelo de vetor de parâmetros $\hat{\beta}$. O Teste de Hausman é realizado sob a hipótese nula de não correlação entre o efeito individual e as variáveis explicativas do modelo (MACHADO et al. 2005).

Se a hipótese nula não for rejeitada, as estimativas por efeitos aleatórios são consistentes e assintoticamente eficientes, e as estimativas por efeitos fixos apenas consistentes e, portanto, se deve escolher o modelo de efeitos aleatórios. Já se a hipótese nula for rejeitada, as estimativas por efeitos aleatórios são inconsistentes e as estimativas por efeitos fixos consistentes, e, assim, deve-se optar pelo modelo de efeitos fixos.

Serão delineados neste estudo as estimações para dados em painel por efeito fixo e por efeito aleatório, bem como os testes de robustez para determinar o melhor modelo.

\section{Especificação do modelo}

$\mathrm{Na}$ análise do comportamento da desigualdade de salários nos municípios brasileiros, foram utilizadas variáveis municipais construídas por meio de informações socioeconômicas dos trabalhadores dos respectivos municípios. Foram estimados quatro modelos com dados em painel, os quais estão especificados na Tabela 01.

Segundo Lee et al. (2016), existe correlação entre o tamanho do município (representado pelo número de vínculos) e a taxa de trabalhadores com nível superior, com o valor do salário médio, uma vez que as grandes cidades e/ou com 
mais residentes altamente qualificados tendem a ter salários mais altos. Além disso, há que se considerar também a relação entre a variável "taxa ganhadores de pico" e o salário médio. Em decorrência dessas relações, algumas variáveis explicativas não foram incluídas em alguns modelos.

Tabela 01: Modelos de relação entre o índice de Gini e as variáveis explicativas

\begin{tabular}{|c|c|}
\hline $\begin{array}{c}\text { Model } \\
0\end{array}$ & Relação Proposta \\
\hline & Modelos incluindo a variável número de vínculos de trabalho \\
\hline 1 & $\ln$ Gin $i=\propto_{i}+\beta_{1}{\ln v i n_{i t}}+c_{i}+u_{i t}$ \\
\hline 2 & $\begin{array}{c}\operatorname{lnGini}=\alpha_{i}+\beta_{1} \text { lnvin }_{i t}+\beta_{2} \text { txtrabfin }_{i t}+\beta_{3} \text { txtrabpub }_{i t}+\beta_{4} \text { txescsup }_{i t}+\beta_{5} \text { txestrange }_{i t} \\
+\beta_{6} \text { txgpico }_{i t}+\beta_{7} \text { txmasc }_{i t}+c_{i}+u_{i t}\end{array}$ \\
\hline & Modelos incluindo a variável salário médio \\
\hline 3 & $\operatorname{lnGini}=\alpha_{i}+\beta_{1}$ lnsalmed $_{i t}+c_{i}+u_{i t}$ \\
\hline 4 & $\begin{aligned} \text { lnGini }= & \alpha_{i}+\beta_{1} \text { lnsalmed }_{i t}+ \\
& +\beta_{2} \text { txtrabfin }_{i t}+\beta_{3} \text { txtrabpub }_{i t}+\beta_{4} \text { txestrange }_{i t}+\beta_{5} \text { txmasc }_{i t}\end{aligned}$ \\
\hline
\end{tabular}

Fonte: Elaboração própria.

Em que InGini corresponde à variável dependente e representa o logaritmo natural do índice de Gini salarial do município. Como variáveis explicativas: Innvin representa o logaritmo natural do número de vínculos ativos no município; Insalmed é o logaritmo natural do salário médio do município; txtrabfin a taxa de trabalhadores no setor financeiro no município; txtrabpub significa a taxa de trabalhadores no setor público no município; txescsup é a taxa de trabalhadores com nível superior no município; txestrange configura a taxa de trabalhadores estrangeiros no município; txgpico é a taxa de ganhadores de pico no município; e txmasc corresponde à taxa de trabalhadores do sexo masculino no município. Os subscritos i e t representam, respectivamente, as $i=1,2, \ldots, 5.555$ unidades observáveis (municípios) e os $t=1$, $2, \ldots, 10$ períodos (anos). Apenas as variáveis Innvin e Insalmed estão em logaritmo natural.

Por fim, caso assuma-se por hipótese que ci é obtido como um processo independente e identicamente distribuído, com média zero e variância $\sigma_{v}^{2}$, então, os modelos representados na Tabela 01 serão estimados com efeitos aleatórios. Caso ci seja um parâmetro fixo, os modelos devem ser estimados com efeitos fixos. 


\section{Resultados e discussão}

Nesta seção serão apresentados os resultados estimados para as equações do índice de Gini salarial em função do tamanho do município, do salário médio e taxas de trabalhadores no setor público, no setor financeiro, daqueles com nível superior, de trabalhadores estrangeiros, de ganhadores de pico e de trabalhadores do sexo masculino, para os municípios brasileiros, nos anos de 2007 a 2016.

Antes de apresentar as estimações dos modelos propostos na pesquisa, decidiu-se, inicialmente, verificar a relação do índice de Gini salarial com o tamanho do município e o salário médio do município, por meio da análise de gráficos de dispersão e tendência.

A Figura 01 contém os gráficos de dispersão e tendência entre o índice de Gini e o logaritmo natural do número de vínculo de trabalho para os municípios brasileiros nos anos de 2007 a 2016.
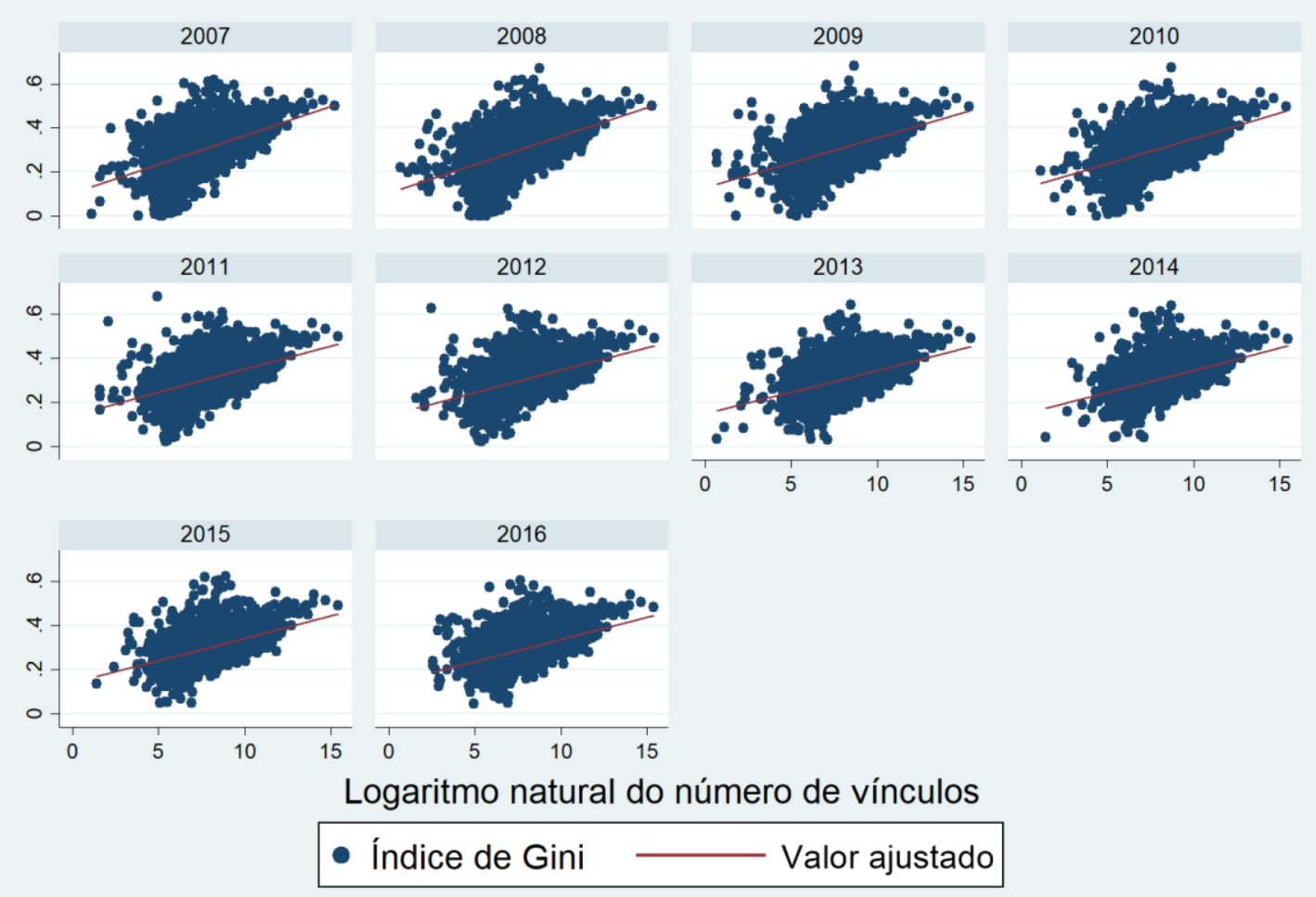

Figura 01: Gráfico de dispersão e tendência do índice de Gini dos municípios brasileiros, de acordo com o logaritmo natural do número de vínculos, por ano.

Fonte: Elaboração própria com dados da RAIS.

Com suporte na análise gráfica, pode-se observar, de maneira geral, a existência de relação positiva entre o índice de Gini salarial e o número de vínculos e, como o número de vínculos é utilizado como proxy para o tamanho da população, 
pode-se sugerir que, quanto maior a população municipal, maior será a desigualdade salarial.

O resultado dessa análise está de acordo com o observado por Korpi (2007), que verificou haver relação positiva entre o tamanho da população e a desigualdade salarial nas cidades suecas. Essa relação também foi observada nos estudos de Glaeser et al. (2009) e Lee et al. (2016), realizados para os Estados Unidos e o Reino Unido, respectivamente.

$\mathrm{Na}$ Figura 02 são expostos os gráficos de dispersão e tendência entre 0 índice de Gini de salários e o logaritmo natural do salário médio para os municípios brasileiros nos anos de 2007 a 2010. É possível observar, com apoio na análise gráfica, relação positiva entre o índice de Gini salarial e o salário médio do município, ou seja, no geral, quanto maior o salário médio, maior será a desigualdade salarial municipal.

A relação positiva observada entre o salário médio e a desigualdade nos municípios brasileiros também está de acordo com a literatura, corroborando os resultados de Korpi (2007), Glaeser et al. (2009) e Lee et al. (2016).
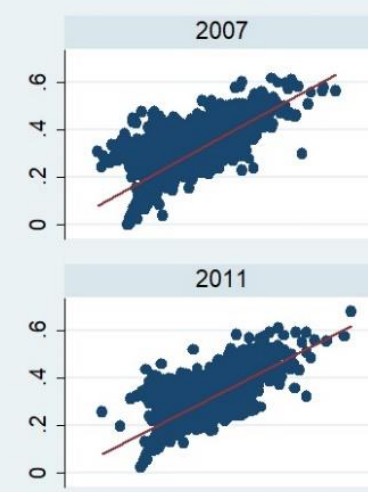

2015

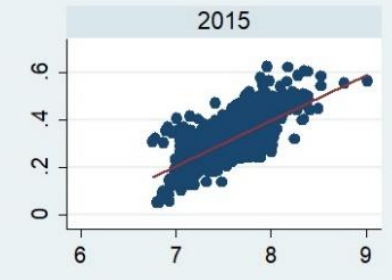

Logaritmo natural do salário médio

- Índice de Gini Valor ajustado

Figura 02: Gráfico de dispersão e tendência do índice de Gini dos municípios brasileiros, de acordo com o logaritmo natural do salário médio, por ano.

Fonte: Elaboração própria com dados da RAIS. 
Os modelos 1, 2, 3 e 4, estimados neste estudo e apresentados nesta parte da pesquisa, estão especificados na Tabela 01. Os resultados estimados dos parâmetros dos modelos 1 e 2, com efeitos fixos e aleatórios, bem como os seus respectivos erros-padrão, são demonstrados na Tabela 02 e, dos modelos 3 e 4, na Tabela 03.

De acordo com o Teste de Hausman, nos quatro modelos estimados, ao nível de $1 \%$ de significância, os melhores resultados são obtidos com regressões por efeitos fixos, ou seja, tais resultados levam à rejeição da hipótese nula de que as diferenças entre os parâmetros estimados pelo método com efeitos fixos e pelo método com efeitos aleatórios não são sistemáticas, aceitando modelo de efeitos fixos, uma vez que os efeitos-controle são estatisticamente correlacionados com os regressores. Sendo assim, serão interpretadas apenas as estimativas por efeitos fixos (Tabelas 02 e 03).

Nos modelos 1 e 2, dispostos na Tabela 02, são expressas as regressões que consideram a variável Innvin, referente ao tamanho da população do munícipio. No primeiro modelo, a regressão é estimada apenas com esta variável, enquanto no segundo são incluídas as demais variáveis explicativas, conforme a especificação dos modelos na Tabela 01. Nos dois modelos expostos na Tabela 02, todos os coeficientes estimados são estatisticamente significantes ao nível de $1 \%$ e apresentaram os sinais esperados, com exceção das variáveis txestrange e txgpico no modelo 2, que se referem, respectivamente, às taxas de trabalhadores estrangeiros e de trabalhadores ganhadores de pico, e não são estatisticamente significantes.

Com relação ao tamanho da população do município, a variável Innvin apresentou sinal positivo em ambas as regressões, ou seja, no Brasil, há relação positiva entre a desigualdade salarial e o tamanho do município, evidenciando o fato de que municípios maiores tendem a apresentar maior nível de desigualdade salarial, o que está de acordo com os resultados de Korpi (2007), Glaeser et al. (2009) e Lee et al. (2016), obtidos respectivamente para o Suécia, Estados Unidos e Reino Unido.

Quando à elasticidade desta variável em relação ao índice de Gini salarial, de acordo com o modelo 1, um aumento de 1\% no tamanho do município resultaria num aumento de $0,032 \%$ na desigualdade salarial. No modelo 2 , com a inclusão das demais variáveis explicativas, o aumento sobre a desigualdade salarial, dado o crescimento de $1 \%$ no tamanho do município, seria de 0,043\%. Observa-se que, ao 
incluir as demais variáveis explicativas na regressão, o impacto do tamanho do município sobre a desigualdade salarial aumenta. Além disso, também se observa aumento no grau de ajustamento do modelo.

Tabela 02: Resultados das estimativas da desigualdade salarial, incluindo a variável número de vínculos de trabalho com dados em painel 2007-2016

\begin{tabular}{|c|c|c|c|c|}
\hline \multirow{2}{*}{$\begin{array}{l}\text { Variáveis } \\
\text { Explicativas }\end{array}$} & \multicolumn{2}{|c|}{ Modelos com Efeitos Fixos } & \multicolumn{2}{|c|}{$\begin{array}{c}\text { Modelos com Efeitos } \\
\text { Aleatórios }\end{array}$} \\
\hline & 1 & 2 & EA1 & EA2 \\
\hline Innvin & $\begin{array}{c}0,032065^{*} \\
(0,00943)\end{array}$ & $\begin{array}{c}0,043362^{*} \\
(0,00959)\end{array}$ & $\begin{array}{l}0,067422^{*} \\
(0,00302)\end{array}$ & $\begin{array}{l}0,069379^{*} \\
(0,00351)\end{array}$ \\
\hline txtrabfin & - & $\begin{array}{c}0,025118^{*} \\
(0,00363)\end{array}$ & - & $\begin{array}{l}0,031098^{*} \\
(0,00347)\end{array}$ \\
\hline txtrabpub & - & $\begin{array}{c}0,001145^{\star} \\
(0,00024)\end{array}$ & - & $\begin{array}{l}0,00079^{*} \\
(0,00021)\end{array}$ \\
\hline txescsup & - & $\begin{array}{l}0,004112^{*} \\
(0,00052)\end{array}$ & - & $\begin{array}{l}0,004218^{*} \\
(0,00043)\end{array}$ \\
\hline txestrange & - & $\begin{array}{c}-0,006574 \\
(0,00497)\end{array}$ & - & $\begin{array}{l}-0,006223 \\
(0,00446)\end{array}$ \\
\hline txgpico & - & $\begin{array}{l}0,001342 \\
(0,00089)\end{array}$ & - & $\begin{array}{l}0,001140 \\
(0,00079)\end{array}$ \\
\hline txmasc & - & $\begin{array}{c}0,0066764^{*} \\
(0,00045)\end{array}$ & - & $\begin{array}{l}0,006415^{*} \\
(0,00038)\end{array}$ \\
\hline Constante & $\begin{array}{l}-1,50225^{*} \\
(0,06755)\end{array}$ & $\begin{array}{l}-2,11334^{*} \\
(0,08637)\end{array}$ & $\begin{array}{l}-1,75551^{*} \\
(0,02221)\end{array}$ & $\begin{array}{l}-2,26692^{*} \\
(0,05135)\end{array}$ \\
\hline $\mathrm{N}$ & 55.550 & 55.550 & 55.550 & 55.550 \\
\hline $\mathrm{R}^{2}$ & 0,1884 & 0,2494 & 0,1884 & 0,2691 \\
\hline $\mathrm{F}$ & $11,56^{*}$ & $45,62^{*}$ & - & - \\
\hline$\chi^{2}$ & - & - & $498,71^{*}$ & $1278,45^{*}$ \\
\hline Hausman & $226.57^{*}$ & 278.61 * & & \\
\hline
\end{tabular}

* significante a $1 \%$; ** significante a $5 \%$.

Fonte: Elaboração própria com dados da RAIS.

No modelo 2, que também considera a taxa de trabalhadores com nível superior (txescsup) para explicar a desigualdade salarial, assim como observado por Korpi (2007) e Lee et al. (2016), pôde-se observar que a taxa de trabalhadores com nível superior se relaciona positivamente com o logaritmo natural do índice de Gini. Verificou-se que, para cada aumento de $1 \%$ na taxa de trabalhadores com nível superior, ocorre aumento de $0,411 \%$ no índice de Gini.

Esse resultado pode parecer contraditório, uma vez que estudos realizados para o Brasil, como os de Miro et al. (2014) e Miro et al. (2016), concluíram que o aumento educacional foi um dos principais fatores que contribuíram para a queda da desigualdade salarial desde o início do século XXI, no entanto, observaram que, 
para quantis de escolaridade superiores, o efeito pode ser contrário, ou seja, dentro da faixa de trabalhadores mais escolarizados, o aumento da escolaridade pode contribuir positivamente com a desigualdade salarial, o que corrobora os resultados deste estudo.

Ainda com relação ao impacto positivo da proporção de trabalhadores com nível superior sobre a desigualdade de salários, não se pode deixar de mencionar o fenômeno "paradoxo do progresso" que, segundo Bourguignon et al. (2005), ocorre quando o progresso educacional pode causar, a priori, o aumento da desigualdade.

O coeficiente estimado da variável txgpico, referente à taxa de ganhadores de pico, de acordo com o modelo 2, não é estatisticamente significante. No estudo de Lee et al. (2016), conquanto esta variável se haja mostrado significante e com sinal positivo para algumas medidas de desigualdade utilizadas por ele, também não apresentou coeficiente estatisticamente significante para explicar variações no índice de Gini.

Na Tabela 03, são apresentadas as equações 3 e 4, especificadas na Tabela 01, que consideram o efeito da variável Insalmed referente ao valor do salário médio do município sobre a desigualdade salarial. Como já enfatizado na Tabela 01, na seção de especificação do modelo, nestas regressões, não são incluídas as variáveis referentes ao tamanho do município, tampouco a taxa de trabalhadores com nível superior e a taxa de ganhadores de pico, em virtude da correlação que estas podem possuir com o valor do salário médio. O modelo 3 é estimado considerando apenas a variável de salário médio. Já no modelo 4, são incluídas as demais variáveis explicativas. Em ambos os modelos, todos os coeficientes estimados são estatisticamente significantes ao nível de 1\% e apresentaram os sinais esperados, exceto a variável que diz respeito à taxa de trabalhadores estrangeiros, não estatisticamente significante.

Os resultados das estimações apontam que, no Brasil, no período analisado, o valor do salário médio do município se relaciona positivamente com o índice de Gini salarial, ou seja, quanto maior o salário médio do município, maior tende a ser o nível de desigualdade salarial nele observado. Esse resultado coincide com os estudos realizados por Korpi (2007), Glaeser et al. (2009) e Lee et al. (2016).

Tabela 03: Resultados das estimativas da desigualdade salarial, incluindo a variável salário médio com dados em painel 2007-2016 


\begin{tabular}{|c|c|c|c|c|}
\hline \multirow[t]{2}{*}{ Explicativas } & & & \multicolumn{2}{|c|}{ Aleatórios } \\
\hline & 3 & 4 & EA3 & EA4 \\
\hline Insalmed & $\begin{array}{l}0,481349^{*} \\
(0,01871)\end{array}$ & $\begin{array}{c}0,503666^{*} \\
(0,01911)\end{array}$ & $\begin{array}{c}0,530654^{*} \\
(0,01605)\end{array}$ & $\begin{array}{l}0,527212^{*} \\
(0,01642)\end{array}$ \\
\hline txtrabfin & & $\begin{array}{c}0,013682^{*} \\
(0,00273)\end{array}$ & $10=0.04$ & $\begin{array}{l}0,016541^{*} \\
(0,00251)\end{array}$ \\
\hline txtrabpub & - & $\begin{array}{l}0,003703^{*} \\
(0,00025)\end{array}$ & - & $\begin{array}{l}0,001438^{*} \\
(0,00013)\end{array}$ \\
\hline txestrange & - & $\begin{array}{l}-0,01959 \\
(0,01111)\end{array}$ & - & $\begin{array}{c}-0,018383^{* *} \\
(0,00942)\end{array}$ \\
\hline txmasc & - & $\begin{array}{c}0,006945^{\star} \\
(0,00042)\end{array}$ & - & $\begin{array}{c}0,004303^{*} \\
(0,00032)\end{array}$ \\
\hline Constante & $\begin{array}{l}-4,79397^{\star} \\
(0,13685)\end{array}$ & $\begin{array}{l}-5,51794^{*} \\
(0,15332) \\
\end{array}$ & $\begin{array}{c}-5,154673^{*} \\
(0,11855) \\
\end{array}$ & $\begin{array}{l}-5,44392^{*} \\
(0,12880)\end{array}$ \\
\hline $\mathrm{N}$ & 55.550 & 55.550 & 55.550 & 55.550 \\
\hline $\mathrm{R}^{2}$ & 0,3988 & 0,2837 & 0,3988 & 0,3851 \\
\hline $\mathrm{F}$ & $662,17^{*}$ & $162,44^{*}$ &  & \\
\hline$\chi^{2}$ & & - & $1093,13^{*}$ & $1165,22^{*}$ \\
\hline Hausman & $539.34^{*}$ & $1537.51^{*}$ & & \\
\hline
\end{tabular}

* significante a 1\%; ${ }^{* *}$ significante a 5\%.

Fonte: Elaboração própria com dados da RAIS.

No que se refere à elasticidade estimada para o valor do salário médio, com relação ao índice de Gini salarial, de acordo com o modelo 3, o aumento de $1 \%$ no valor do salário médio resultaria no aumento de $0,481 \%$ na desigualdade salarial. Já no modelo 4 , considerando as demais variáveis explicativas, o aumento de $1 \%$ no valor do salário médio resultaria no crescimento de $0,503 \%$ na desigualdade salarial. Este resultado pode ser justificado pelo fato de que a média salarial mais alta pode ser associada à ocorrência de trabalhadores no município com salários muito elevados, o que eleva a média salarial mesmo com existência de salários baixos.

No que diz respeito às variáveis relativas aos setores de trabalho incluídas nos modelos 2 e 4 (tabelas 02 e 03), como se esperava, as taxas de trabalhadores no setor financeiro (txtrabfin) e de trabalhadores no setor público (txtrabpub), demonstraram relação positiva com o logaritmo natural do índice de Gini, isto é, quanto maior a proporção de trabalhadores empregados nesses setores do mercado no município, mais desiguais tendem a ser os salários pagos.

De acordo com o modelo 2, o aumento de 1\% na taxa de trabalhadores do setor financeiro resulta em uma elevação de $2,511 \%$ na desigualdade salarial. Já de acordo com o modelo 4, essa elevação é de 1,368\%. Lee et al. (2016) observaram resultado similar em seu estudo para as cidades do Reino Unido. 
Quanto à taxa de trabalhadores empregados no setor público, conforme o modelo 2, o aumento de $1 \%$ nesta variável resulta em um aumento de $0,114 \%$ na desigualdade salarial. Já de acordo com o modelo 4, esse aumento é de 0,37\%. Uma das explicações para este resultado é o diferencial de salários entre o setor público e o privado, em que, segundo Vaz e Roffmann (2006) e Souza e Medeiros (2013), se observa o pagamento de salários mais elevados para funcionários públicos do que para empregados do setor privado que possuem atributos semelhantes e realizam as mesmas atividades.

Conforme pode ser visto nas tabelas 02 e 03, a variável taxa de trabalhadores estrangeiros (txestrange) não é estatisticamente significante em ambos os modelos nos quais foi incluída (modelos 2 e 4). Esse resultado, de certo modo, já era esperado, haja vista a pouca expressividade da população estrangeira na maioria dos municípios brasileiros.

Por último, como pode ser visto nos modelos 2 e 4 (tabelas 02 e 03), a taxa de trabalhadores do sexo masculino (txmasc) também apresenta relação positiva com a desigualdade salarial, coincidindo com o estudo de Korpi (2007), no qual concluiu que quanto maior a participação masculina na força de trabalho de determinado local, maior tende a ser o grau de desigualdade salarial ali observado. De acordo com o modelo 2, para cada aumento de 1\% na taxa de trabalhadores do sexo masculino, há um aumento de $0,667 \%$ no índice de Gini. Já conforme o modelo 4 , esse aumento seria de $0,694 \%$.

\section{Considerações Finais}

Esta pesquisa teve como objetivo analisar a relação entre o nível de desigualdade salarial municipal e o tamanho populacional do município, bem como o valor do salário médio do município, além de outros condicionantes, com suporte em dados anuais, no período de 2007 a 2016, utilizando uma amostra de 5.555 municípios brasileiros.

Com efeito, o estudo ora relatado cobre uma lacuna importante da literatura, ao tratar da questão da desigualdade salarial ao nível de municípios, como preconizam autores como Glaeser et al. (2009) e Lee et al. (2016).

No geral, as principais hipóteses do estudo foram confirmadas, haja vista que se constatou, no Brasil, a existência de relação positiva entre a desigualdade salarial e o tamanho da população do município, bem como com o valor do salário médio do município, em acordo com a literatura internacional. 
Confirma-se também, a influência favorável à desigualdade salarial, nos municípios brasileiros, de fatores como: taxa de trabalhadores no setor financeiro, taxa de trabalhadores no setor público, taxa de trabalhadores com nível superior e taxa de trabalhadores do sexo masculino.

Em suma, foi possível observar que a boa parte da desigualdade de salários é gerada pela existência dos diferenciais salariais entre setores de trabalho (percentual de participação no setor público e financeiro), classe de escolaridade (educação superior) e sexo do trabalhador.

Sugere-se, assim, às distintas escalas de governo (federal, estadual e municipal), que, na adoção de políticas públicas que tenham como objetivo a diminuição da desigualdade salarial, sejam observados tais efeitos, ou seja, há que se combater os diferenciais de salários por setor de trabalho, classe de escolaridade e sexo do trabalhador, a fim de se contribuir para melhor distribuição da renda do trabalho no País.

\section{REFERÊNCIAS}

ASSIS, R. S.; ALVES, J. S. Hiato salarial entre homens e mulheres no Brasil segundo condição migratória: o mercado de trabalho é segregado ou discrimina?. Revista

Econômica do Nordeste, Fortaleza, v. 45, n. 1, p. 120-135, 2014.

BALASSIANO, M.; SEABRA, A. A.; LEMOS, A. H. Escolaridade, salários e empregabilidade: tem razão a Teoria do Capital Humano?. Revista de Administração Contemporânea, v. 9, n. 4, p. 31-52, 2005.

BALTAGI, B. H. Econometric analysis of panel data. 3. ed. John Wiley \& Sons Ltd. 2005. BARROS, R. P.; CARVALHO, M.; FRANCO, S.; MENDONÇA, R. Conseqüências e causas imediatas da queda recente da desigualdade de renda brasileira. Rio de Janeiro, IPEA, 2006. (Texto para Discussão, n. 1201).

BARROS, R. P.; FRANCO, S.; MENDONÇA, R. A recente queda da desigualdade de renda e o acelerado progresso educacional brasileiro da última década. Rio de Janeiro, IPEA, 2007. (Texto para Discussão, n. 1304).

BARROS, R.; REIS, J. G. A. Wage inequality and the distribution of education: a study of the evolution of the regional differences in inequality in metropolitan Brazil. Journal of Development Economics, n. 36, p. 117-143, 1991.

BATISTA, N. N. F.; CACCIAMALI, M. C. Diferencial de salários entre homens e mulheres segundo a condição de migração. Revista Brasileira de Estudos Populacionais, Rio de Janeiro, v. 26, n. 1, p. 97-115, 2009.

BAUM-SNOW, N.; PAVAN, R. Inequality and city size. Review of Economics and Statistics, v. 95, n. 5, p. 1535-1548, 2013.

BEHRENS, K.; ROBERT-NICOULD, F. Survival of the fittest in cities: Agglomeration, selection, and polarisation. CEP Discussion Paper, n. 894., Oct. 2008. BOURGUIGNON, F.; FERREIRA, F. H. G.; LUSTIG, N. The microeconomics of income distribution dynamics in East Asia and Latin America. Washington, DC: World Bank and Oxford University Press, 2005.

BUDRÍA, S.; TELHADO-PEREIRA, P. Educational qualifications and wage inequality: evidence for Europe. Revista de Economía Aplicada, v. 19, n. 56, p. 5-34, 2011. 
CHAVES, A. L. L. Determinação dos rendimentos na Região Metropolitana de Porto Alegre: uma verificação empírica da Teoria do Capital Humano. Ensaios FEE, Porto Alegre, v. 23, p. 399-420, 2002. Edição especial.

DUCLOS, J. Y.; ESTEBAN, J.; RAY, D. Polarization: concepts, measurement, estimation.

Econometrica, v. 72, n. 6, p. 1737-1772, 2004.

FERREIRA, F. H. G.; LEITE, P. G.; LITCHFIELD, J. A. The rise and fall of Brazilian Inequality: 1981-2004. Macroeconomic Dynamics, Cambridge University Press, v. 12, p. 199-230, 2008.

GLAESER, E. L.; RESSEGER, M.; TOBIO, K. Inequality in cities. Journal of Regional Science, v. 49, n. 4, p. 617-646, 2009.

GREENE, W. H. Econometric analysis. 4. ed. New Jersey: Prentice Hall, 2000.

HOFFMANN, R. Distribuição de renda e crescimento econômico. Estudos Avançados, São Paulo, v. 15, n. 41, p. 67-76, 2001.]

HOFFMANN, R. Transferências de renda e a redução da desigualdade no Brasil e cinco regiões entre 1997 e 2004. Revista Econômica, Rio de Janeiro, v. 8, n. 1, 2006.

HSIAO, C. Analysis of panel data (Econometric Society Monographs). 2. ed. Cambridge University Press, 2003.

IBGE - INSTITUTO BRASILEIRO DE GEOGRAFIA E ESTATÍSTICA. Disponível em $<\mathrm{http}: / /$ www.ibge.gov.br>. Acesso em: 07 de março de 2018.

IBGE. Pesquisa Nacional por Amostra de Domicílios (microdados). Rio de Janeiro: 2014. IPEA. Sobre a Recente Queda da Desigualdade de Renda no Brasil. [S.I.], 2006. Nota técnica. Disponível em:

<http://ipea.gov.br/agencia/images/stories/PDFs/2006_nt01_agosto_disoc.pdf>. Acesso em: dez. 2017.

KORPI, M. Does size of local labour markets affect wage inequality? A rank-size rule of income distribution. Journal of Economic Geography, v. 8, n. 2, p. 211-237, 2007.

KUZNETS, S. Economic growth and income inequality. The American Economic Review, v. 45, n. 1, p. 1-28, 1955.

LAVINAS, L.; NICOLL, M. Pobreza, transferências de renda e desigualdades de gênero: conexões diversas. Parcerias estratégicas, v. 11, n. 22, p. 39-76, 2006.

LEE, N.; SISSONS, P.; JONES, K. The geography of wage inequality in British cities.

Regional Studies, v. 50, n. 10, p. 1714-1727, 2016.

LEMIEUX, T. Post-secondary education and increasing wage inequality. The American

Economic Review, v. 96, n. 2, p. 195-199, 2006.

MACHADO, J. B. B.; SACHSIDA, A.; MENDONÇA, M. J. C. Abertura Comercial e Inflação:

Uma Análise para Dados em Painel. Estudos Econômicos, São Paulo, v. 35, n.3, p. 547-

567, 2005.

MARQUES JUNIOR, K. A renda, desigualdade e criminalidade no Brasil: uma análise empírica. Revista Econômica do Nordeste, Fortaleza, v. 45, n. 1, p. 34-46, jan./mar. 2014.

MIRO, V. H.; FRANÇA, J. M. S.; PINHO NETO, V. R. Capital humano e desigualdade salarial no Brasil: uma análise de decomposição para o período 1995-2014. Estudos

Econômicos, São Paulo, v. 46, n. 3, p. 579-608, 2016.

MIRO, V. H.; FRANÇA, J. M. S.; PINHO NETO, V. R. Capital humano e desigualdade salarial no Brasil: uma análise de decomposição para o período 2001-2012. In: ENCONTRO NACIONAL DE ECONOMIA, 42., 2014, Natal/ RN. Anais... Natal: $42^{\circ}$ ENCONTRO NACIONAL DE ECONOMIA, 2014.

MIRO, V. H.; SULIANO, D. C. Discriminação de rendimentos por gênero e raça a partir de realidades sócio-econômicas distintas. In: CARVALHO, E. B. S. et al. (Org.). Economia do Ceará em Debate 2009. 5. ed. Fortaleza: IPECE, 2010, p. 36-56.

RAMOS, L. Desigualdade de Rendimentos do Trabalho no Brasil de 1995 a 2005. In: BARROS, R. P.; FOGUEL, M. N.; ULYSSEA, G. (Org.). Desigualdade de Renda no Brasil: uma análise da queda recente. Brasília: Ipea, 2007. v. 2, p. 267-284.

RESENDE, J. P.; ANDRADE, M. V. Crime social, castigo social: desigualdade de renda e taxas de criminalidade nos grandes municípios brasileiros. Estudos Econômicos, São Paulo, v. 41, n. 1, p. 173-195, 2011. 
SANTOS, E. S.; CAMILLO, V. S. Capital humano e renda do trabalho no Brasil: uma investigação empírica. In: CICLO DE DEBATES EM ECONOMIA INDUSTRIAL, TRABALHO E TECNOLOGIA, 9., 2011, São Paulo. Anais... São Paulo: PUC/SP, 2011.

SONAGLIO, C. M.; ZAMBERLAN, C. O.; LIMA, J. E.; CAMPOS, A. C. Evidências de desindustrialização no Brasil: uma análise com dados em painel. Economia Aplicada, Ribeirão Preto, v. 14, n. 4, p. 347-372, 2010.

SOUZA, P. H. G. F.; MEDEIROS, M. Diferencial salarial público-privado e desigualdade de renda per capita no Brasil. Estudos Econômicos, São Paulo, v. 43, n. 1, p. 5-28, 2013. SZWARCWALD, C. L.; BASTOS, F. I.; ESTEVES, M. A. P.; ANDRADE, C. L. T.; PAEZ, M. S.; MEDICI, E. V.; DERRICO, M. Desigualdade de renda e situação de saúde: o caso do Rio de Janeiro. Cad. Saúde Pública, Rio de Janeiro, v. 15, n. 1, p.15-28, 1999.

VAZ, D. V.; HOFFMANN, R. Remuneração nos serviços no Brasil: o contraste entre funcionários públicos e privados. Economia e Sociedade, Campinas, v. 16, n. 2, p. 199232, 2007.

WOOLDRIDGE, Jeffrey M. Econometric Analysis of Cross Section and Panel Data. The MIT Press, Cambridge, MA, 2002.

\section{NOTAS DE AUTOR}

\section{CONTRIBUIÇÃO DE AUTORIA}

Matheus Oliveira de Alencar - Concepção. Coleta de dados. Análise de dados. Elaboração do manuscrito. Revisão e aprovação da versão final do trabalho.

Jair Andrade Araújo - Concepção. Elaboração do manuscrito. Participação ativa da discussão dos resultados. Revisão e aprovação da versão final do trabalho.

Wellington Ribeiro Justo - Concepção. Elaboração do manuscrito. Participação ativa da discussão dos resultados. Revisão e aprovação da versão final do trabalho.

\section{FINANCIAMENTO}

Apoio financeiro do Conselho Nacional de Desenvolvimento Científico e Tecnológico (CNPq) na forma de bolsa.

\section{CONSENTIMENTO DE USO DE IMAGEM}

Não se aplica.

\section{APROVAÇÃO DE COMITÊ DE ÉTICA EM PESQUISA}

Não se aplica.

\section{CONFLITO DE INTERESSES}

Não se aplica.

\section{LICENÇA DE USO}

Este artigo está licenciado sob a Licença Creative Commons CC-BY. Com essa licença você pode compartilhar, adaptar, criar para qualquer fim, desde que atribua a autoria da obra.

\section{HISTÓRICO}

Recebido em: 20-11-2018

Aprovado em: 24-06-2019 